\title{
Basic Research Advances on Pituitary Stem Cell Function and Regulation
}

\author{
John P. Russell Emily J. Lodge Cynthia L. Andoniadou \\ Centre for Craniofacial and Regenerative Biology, King's College London, London, UK
}

\section{Keywords}

Pituitary stem cell · SOX2 · YAP/TAZ · Hippo · Regenerative medicine

\begin{abstract}
As a central regulator of major physiological processes, the pituitary gland is a highly dynamic organ, capable of responding to hormonal demand and hypothalamic influence, through adapting secretion as well as remodelling cell numbers among its seven populations of differentiated cells. Stem cells of the pituitary have been shown to actively generate new cells during postnatal development but remain mostly quiescent during adulthood, where they persist as a long-lived population. Despite a significant body of research characterising attributes of anterior pituitary stem cells, the regulation of this population is poorly understood. A better grasp on the signalling mechanisms influencing stem proliferation and cell fate decisions can impact on our future treatments of pituitary gland disorders such as organ failure and pituitary tumours, which can disrupt endocrine homeostasis with life-long consequences. This minireview addresses the current methodologies aiming to understand better the attributes of pituitary stem cells and the normal regulation of this population in the organ, and discusses putative future avenues to manipulate pituitary stem cells during disease states or regenerative medicine approaches.
\end{abstract}

(c) 2018 S. Karger AG, Basel

\section{KARGER}

(C) 2018 S. Karger AG, Basel

E-Mail karger@karger.com

www.karger.com/nen

\section{Introduction}

Stem cells are characterised by their ability to selfrenew and to give rise to differentiated cells, and fulfil an important function in the generation of new cells during growth, homeostasis, and repair. Resident stem cell contribution to normal postnatal homeostasis has been demonstrated in the murine pituitary [1], a step ahead of other endocrine organs of great interest to regenerative medicine, such as the pancreas [2]. Despite the presence of these stem cells, the adult pituitary shows limited regenerative capacity to recover from insult, in either mouse models or human conditions. Endogenous pituitary stem cells display a decline in their potential with age [3], and recent data suggest that their participation in homeostasis during insult is minimal [4]. However, certain attributes of endocrine cells allow them to contribute to enhancing function during insult and enable a degree of resilience to the anterior pituitary. These attributes include: (1) phenotypic plasticity/the possibility to transdifferentiate when a dynamic shift in the population is required, for instance, from somatotrophs to lactotrophs as indicated by bihormonal expression during regeneration [5]; (2) proliferative capacity among the hormone-expressing cell populations; (3) the ability of reduced numbers of endocrine cells to compensate for hormone production after insult (e.g., ablation of even up to $80 \%$ of somato-

Cynthia L. Andoniadou

Centre for Craniofacial and Regenerative Biology, King's College London

Floor 27 Tower Wing, Guy's Campus

London SE1 9RT (UK)

E-Mail cynthia.andoniadou@ kcl.ac.uk 
trophs in mice does not impact on IGF1 levels; however, when ablation reaches a threshold of $90 \%$ there is a reduction in IGF1) [6]. Combined, these mechanisms may help the organ cope with fluctuations in physiological demand but are clearly not sufficient during insult.

A whole host of markers have been identified for pituitary stem cells; yet the functional relevance of most of them still remains elusive. Consequently, no solid steps have been made in regulating pituitary stem cell proliferation in a controlled manner, or directing their differentiation in vivo. This may be achievable by understanding and manipulating the signalling cues that normally regulate cell activity and fate decisions. Collectively, this information would be applicable to controlling endogenous populations of cells in a broader range of pituitary disorders spanning hypopituitarism and the uncontrolled proliferation of cells during neoplasia, as well as potentially improving hormonal function during ageing.

Recent novel technical advances are enabling us to gain exponential amounts of knowledge about the in vivo cellular regulation and function of the pituitary. Genomic, spatial, and functional information from basic research efforts will need to be integrated and contextualised in order to better inform translation into the clinic. In this review we present an overview of current basic approaches focusing on understanding the endogenous stem cell population of the pituitary.

\section{Generation of Pituitary Endocrine Cells from Pluripotent Stem Cells}

Current regenerative approaches aiming to tackle disorders such as hypopituitarism rely heavily on understanding the characteristics of normal embryonic development and pituitary fate commitment. The lack of human pituitary cell lines has been a hindrance to understanding the key steps regulating patterning and lineage restriction. In recent years, pluripotent stem cells have aided in this process: through sequential treatment with signalling molecules, they can be differentiated to eventually specify a pituitary primordium. Pioneering proof-ofprinciple studies have demonstrated that in this way it is possible to obtain fully functioning hormone-secreting cells in vitro, albeit inefficiently, and to even generate these from human embryonic stem cells following directed differentiation [7-9].

In previous reports, the generation of anterior pituitary fates from embryonic stem cells in three-dimensional culture has been shown to necessitate an intermediate induction to forebrain fates. Specification of oral ectoderm is achieved through treatment with BMP4, and subsequently $\mathrm{SHH}$ agonists to generate the definitive anterior pituitary. This occurs at a low efficiency, where the majority of the cells remain neural and only a small fraction of the non-neural cells (1-7\%) express hormones [10]. A higher but still suboptimal efficiency was obtained in monolayer cultures with human induced pluripotent stem cells. This method employed inhibitors of BMP signalling together with inhibitors of TGF- $\beta$, activin and nodal [9]. The key to this was timed removal of BMP inhibition 2 days after neural induction, leading to placode induction, followed by activation of the SHH signalling pathway to induce oral ectoderm fates. Earlier exposure to BMP4 can increase the overall placode yield [11].

Recent studies demonstrated a derivation of anterior pituitary hormone-producing cells from human embryonic or induced pluripotent stem cells, which are able to function in response to stimulus in murine hypopituitarism models $[8,12]$, with the two-dimensional approach by Zimmer et al. [12] generating functional cells at higher efficiency. In order for in vitro-generated cells to achieve homeostatic control in vivo, these have to be grafted within the pituitary or in immediate contact with the hypothalamus [13-16]. Alternative grafts, such as under the kidney capsule, can function without hypothalamic regulation $[5,10]$, but they have the advantage of being less invasive and more easily accessible.

It is important to note that regeneration of pituitary cell types would not be a viable strategy to treat hypothalamic hypopituitarism. However, transplantation approaches placing new cells in immediate contact with the hypothalamus may partly circumvent hypopituitarism in the case of pituitary stalk interruption or following traumatic brain injury. Future improvements in these protocols and confirmation of functional hormone-producing cells from induced pluripotent stem cells will bring us closer to patient-tailored cell replacement therapies.

\section{Pituitary Stem Cells Are Contained within the SOX2+ Population}

The anterior pituitary is a medium- to low-turnover organ, where a proliferative potential and contribution to all anterior pituitary lineages in the postnatal period of the mouse have been demonstrated by cells expressing SOX2 [1]. This transcription factor serves as a marker of numerous other adult stem cell populations [17], and is also a core factor required for pluripotency of the early 
Fig. 1. Immunofluorescence staining against SOX2 in aged human pituitaries. SOX2 is expressed in cells of the parenchyma of two post-mortem pituitaries (pituitary 1 - female, 77 years; pituitary 2 - male, 89 years). Nuclear localisation is observed in individual parenchymal cells (arrowheads). The nuclei are counterstained with DAPI.

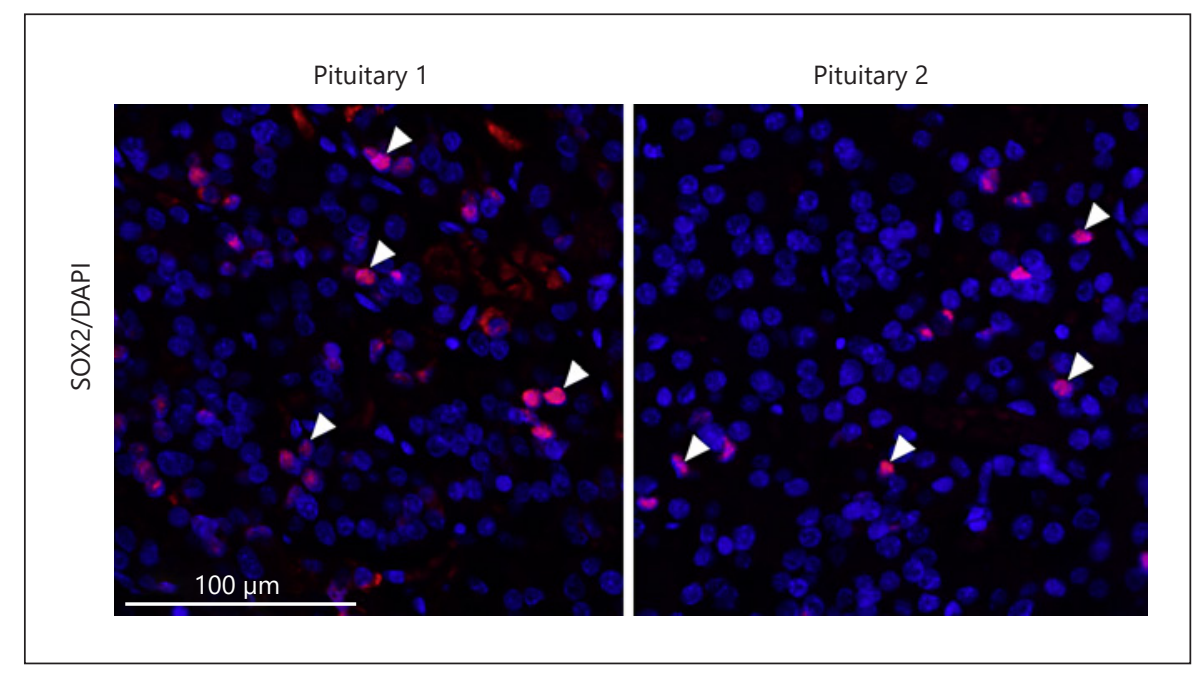

embryo, embryonic stem cells, and reprogramming. The anterior pituitary forms from Rathke's pouch, a primordium in the oral epithelium, where all cells initially express and require SOX2 [18]. By postnatal stages, the positive cells are located in two niches: in the epithelium of the marginal zone and scattered throughout the parenchyma, mostly clustered in small groups. These niches persist throughout life and are interconnected by long cellular processes $[19,20]$.

Our previous work identified that this long-lived population can contribute new hormone-secreting cell types during postnatal life [1], just as it does during organogenesis [21]. Whilst the measure of self-renewal has been limited to in vitro experiments [22], the potential to selfrenew lies only within a proportion of the SOX2 population, under defined stem cell-promoting conditions [23]. In this context, the proportion of SOX 2 cells that adhere expands to generate more SOX2 cells. This does not mean that other cell types in the pituitary, such as subsets of the committed lineages, do not have self-renewal capabilities, and this remains to be tested. It is not known if all SOX2+ cells have the same capacity; it is possible that during in vitro isolation, only cells perceiving particular extrinsic or intrinsic signals, or being in a specific phase of the cell cycle, are capable of activation and divide under these defined conditions. Intrinsic heterogeneity occurring within this SOX2 population, or influences from their in vivo niche, may render them "active or inactive." Of relevance, SOX2 is expressed in the human pituitary in a pattern similar to that of rodents [24], and our data indicate that just like in model systems, this putative stem cell population persists until late adult life (Fig. 1). Therefore, a thor- ough characterisation of this long-lived population would be of value to regenerative medicine approaches. Cells with clonogenic potential have been isolated from normal human pituitaries, and these stem-like cells demonstrated generation of differentiated, hormone-secreting cells [25].

Multiple, non-exclusively overlapping markers have been described in Sox2-expressing cells, such as S100b, Gfra2, Sox9, Nestin, Cdh1, Prop1, Prrx1/2, Cxadr, and Grhl2 [1, 21, 22, 26-31]; however, currently there are no functional data for a requirement of these in the maintenance and promotion of the postnatal stem cell state. The localisation of PROP1 marks a proportion of SOX2+ cells that are able to form colonies in vitro, but this was shown to be required for enabling transition to the progenitor state and for leaving the epithelium via epithelial-mesenchymal transition [32]. SOX9+ cells (also expressing SOX2) do generate functional cells of all lineages in vivo [21], but the difference in potential between single SOX2+ only and double SOX2;SOX9 is not yet known, neither is the functional requirement for SOX9.

\section{Addressing the Heterogeneity of Pituitary Stem Cells}

One thing missing from the current analyses and descriptive characterisations of the stem cell population is the extent of heterogeneity displayed by these cells in their functional attributes. In terms of gene expression, this can now be determined by single-cell RNA-Seq approaches [33]. With advances in cell separation and the depth of sequencing, several thousand cells can be se- 
quenced simultaneously; however, spatial information is lost with these techniques. Methodologies for RNA sequencing in situ are gaining in their cellular resolution and are efficiently analysed; therefore, spatial transcriptomic analyses may be the best methodologies for understanding heterogeneity in a niche-dependent context [34-36].

Such approaches are timely and will reveal a wealth of information, but hypotheses will need to be functionally tested. It is unlikely that SOX2+ cells all have the same capacity in vivo, especially given their differential in vitro potential, heterogeneous marker expression, and differential localisation in vivo. Therefore, we may be able to elucidate in future whether true multipotent $\mathrm{SOX} 2+$ stem cells remain in the adult gland, able to self-renew and give rise to all committed lineages positive for POU1F1 (PIT1), TBX19 (TPIT), and NR5A1 (SF1)/GATA2, or whether SOX2+ cells experience fate restriction, resulting in different uni- or bipotent SOX2-expressing populations. It will also be interesting to determine the localisation of the more active stem cells in order to decipher the particulars of the niches promoting or enabling this state.

Simultaneously addressing the in vivo behaviour of multiple cell types is not trivial. The most appropriate current genetic tools include multicolour reporters [37]. The commonly used Cre recombinase-dependent Confetti reporter [38], for example, labels cells driving Cre and their descendants in any of four fluorescent colours in heterozygosity (Fig. 2a) and 16 distinct combinations in homozygosity. These allow the differential labelling of neighbouring cells in the tissue and an independent study of their fates. Caveats regarding these reporters include: (1) a lower recombination efficiency compared to other single-colour ROSA26 reporters, (2) that recombination can sometimes take place as such where no fluorescent protein is expressed (failure to excise the stop cassette), and (3) the fact that whilst Cre recombinase is expressed in a cell, the expression of a colour can keep changing until Cre is no longer active. In our use of Hes $x 1^{\mathrm{Crel} /+}$; $R 26^{\text {Confetti/+ }}$, by 15.5 days post coitum each labelled cell has a final colour since Hes $x 1$ is no longer expressed, revealing a mixed pattern of closely, non-clonally labelled cells. By 3 months after birth, we find a pattern of frequently segregated colour labelling spanning all fluorophores, indicative of clonal expansion, as well as areas of broad expansion along the epithelium and into the deeper parenchymal layers.

Although the use of this reporter in the embryonic model may not be suitable for analyses of the potential of single cells, it is of value to help us understand cell dynam- ics during growth and to calculate cell turnover in this organ. The Confetti reporter is ideal, however, for inducible systems (e.g., CreERT2), where cells become labelled only whilst the administered tamoxifen is still in the system. One issue is that they ought to be optimised for each inducible driver strain. It is advisable to carry out "nearest neighbour" analyses at short pulses following a titred tamoxifen dosage to define how often two cells next to each other might be labelled with the same colour. When trying to determine if a SOX2+ pituitary stem cell can be multipotent in adulthood or not, this becomes crucial. An example of its optimised use for clonality in the SOX2+ cells of the anterior lobe during the postnatal growth phase $[39,40]$ demonstrates asymmetric divisions along the marginal zone epithelium 2 weeks after induction (Fig. 2b). Deciphering the extent of pituitary stem cell heterogeneity by combining in silico and functional techniques will help determine the characteristics and possible distinct roles of cell subsets within the stem cell population.

\section{Signalling Pathways in the Control of Proliferative Function}

We have previously characterised a drop in stem cell potential with age by determining the in vitro capacity of anterior pituitary cells to form clonal colonies [3]. Similarly, the regenerative capacity of the gland following injury has been reported to decline with age [41]. At any stage of life, no more than one-twentieth of the SOX2 cells are dividing in vivo, or can actively expand in vitro $[1,3]$. Experiments employing physiological challenges do suggest that the SOX2 population can be minimally reactivated; for example, oestradiol treatment instigates proliferation in the SOX2 compartment, increasing the proportion of dividing cells up to $10 \%$ in adulthood [21], a time when divisions are dwindling. Even after a challenge, however, stem cell reactivation remains curiously low and is not the main source of new hormone-secreting cells. Might it therefore be better to focus efforts on more committed cell types that display more proliferative potential? Following adrenalectomy, only up to a fifth of newly generated corticotrophs derive from stem cells [21], and elegant genetic experiments have demonstrated that the majority derive from non-stem cells, either by expansion of committed progenitors or hormone-secreting cells [42]. Lactotrophs can also be stimulated to proliferate by exogenous administration of oestradiol, promoting proliferation both directly and via hypothalamic 


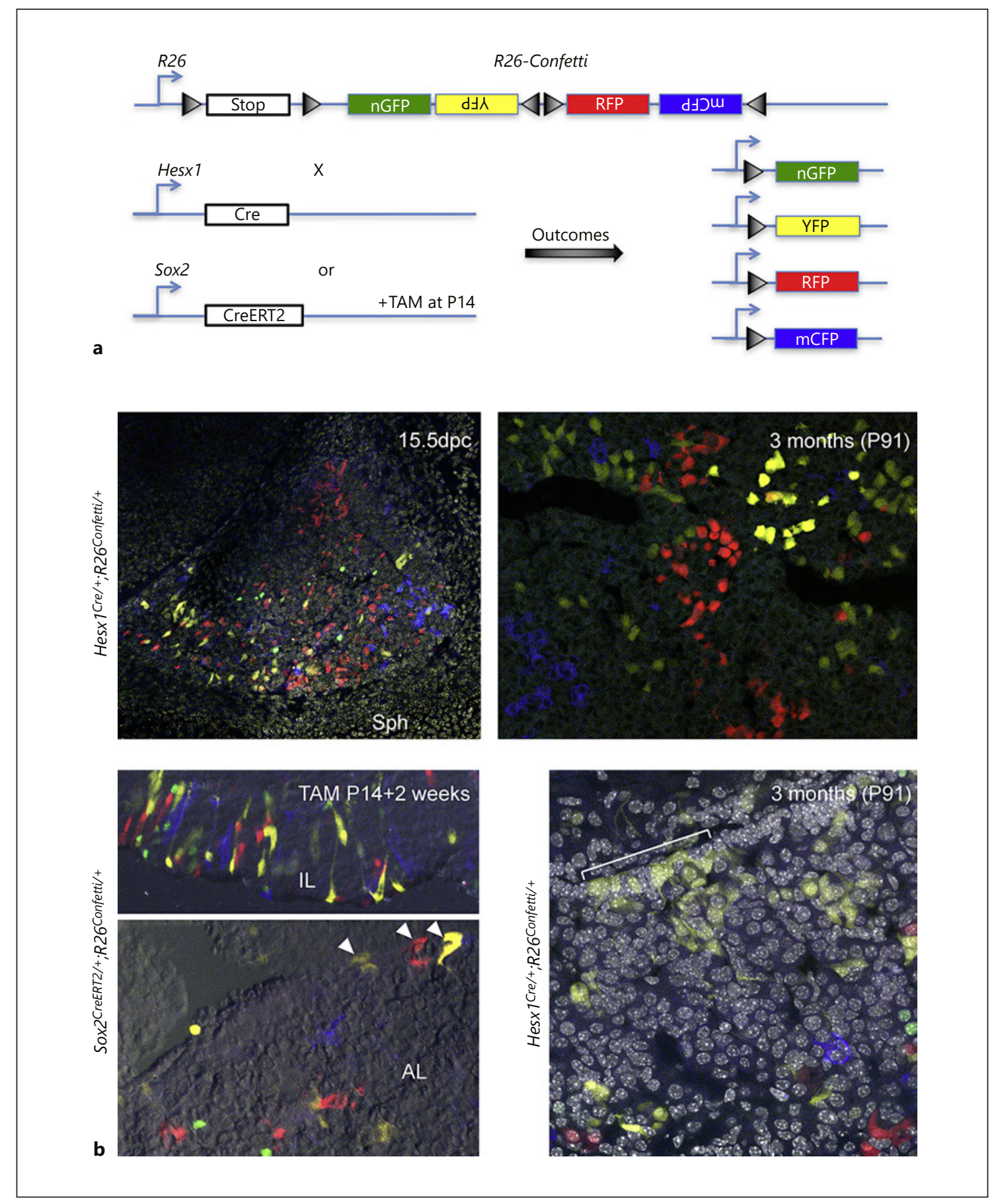

Fig. 2. Multicolour labelling of embryonic and postnatal progenitor/stem cells in the murine pituitary. a Genetic strategy employing the R26-Confetti strain, crossed with either a Cre strain driven from the Hes $x 1$ promoter (embryonic), or a CreERT2 strain driven by the Sox 2 promoter (conditional upon tamoxifen administration). Recombination can result in the stable expression of one of four different fluorophores (nuclear green fluorescent protein [nGFP], yellow fluorescent protein [YFP], red fluorescent protein $[\mathrm{RFP}]$, and membrane cyan fluorescent protein [mCFP]), which continue to be expressed by descendants. b Recombination in the developing pituitary of $\operatorname{Hes} \times 1^{\mathrm{Cre} /+} ; \mathrm{R}_{2} 6^{\mathrm{Confetti} /+}$ demonstrating labelling of numerous scattered cells at 15.5 days post coitum (dpc), followed by analysis at 3 months postnatally, revealing singlecolour clonal expansion of individual embryonic progenitors. A region of expansion (bracket, yellow cells) can be seen spanning the epithelium as well as expanding into the parenchyma. Induction of Sox $2^{\mathrm{CreERT2/+}} ; \mathrm{R} 26^{\mathrm{Confetti} /+}$ pituitaries at postnatal day (P) 14 and analysis 2 weeks later demonstrate labelling of scattered cells in the intermediate lobe (IL) and anterior lobe (AL) as well as sporadic duplication of labelled cells along the epithelium (arrowheads). 


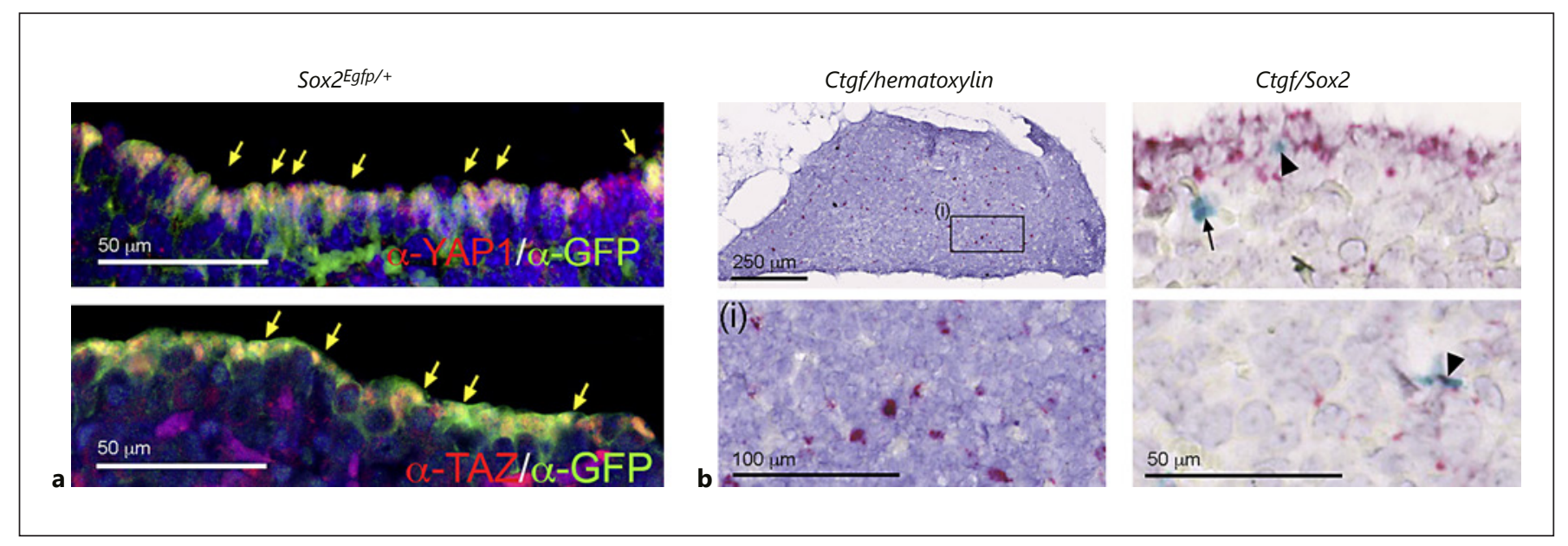

Fig. 3. YAP/TAZ and target expression in the SOX2+ population of pituitary stem cells. a YAP and TAZ localise in the nucleus of SOX2+ cells, marked by green fluorescent protein (GFP) expression in Sox $2^{\text {Egfp/+ }}$ knock-in mice. Adapted from Lodge et al. [51]. b Left: RNAscope mRNA in situ hybridisation detecting that the YAP/TAZ target Ctgf reveals sporadic robust expression in paren- chymal cells (red) and rare expression along the marginal zone epithelium. The cells are counterstained with haematoxylin. Right: double in situ hybridisation detecting Ctgf (aqua, arrow) and Sox2 (red) reveals a partial overlap among Ctgf-expressing cells (arrowheads), and that the majority of Sox2-expressing cells do not express Ctgf. feedback [43]. These properties may be indicative of a large proportion of hormone-expressing cells that are not actually terminally differentiated, or may not have exited the cell cycle whilst functioning by secreting hormone.

The regenerative potential can be influenced by enhancement of signalling pathways promoting proliferation. Forced activation of the WNT pathway in SOX2 cells instigates a burst of proliferative activity [1], in keeping with the major property of this pathway to promote stem cell self-renewal [44]. We have shown that these cells, expressing a stable mutant form of CTNNB1 ( $\beta$-catenin), in turn upregulate the expression of genes from the Wnt, Fgf, Egf, Bmp, Tgf, and $H h$ families [23], all with the potential to influence proliferation and/or cell fate specification. Whether normal postnatal SOX2+ stem cells secrete any of the signals influencing surrounding cells remains to be shown; it would provide an additional function for the relatively sedentary stem cell population in this organ. The outcome of simultaneous enhanced activation of these signalling pathways from mutant SOX2 cells is the paracrine formation of tumours by a massive proliferative response in non-mutant cells, which happen to lie outside of the Sox2 lineage [1].

Interestingly, when committed anterior pituitary cells are targeted to express the same non-degradable mutant form of $\beta$-catenin, they do not generate tumours. Recent work reveals that the tumours form when mutant cells enter senescence and robustly activate the senescenceassociated secretory pathway (SASP) [45]. The SASP response leads to secretion of a range of mitogens, chemokines, and cytokines from the mutated stem cells. In mouse models where the SASP response is low (e.g., through induction of a different mutation elevating the WNT pathway through the Apc gene, or mutating Ctnnb1 in ageing pituitary stem cells), tumours do not form. This might suggest that even if committed cells did initiate the SASP response, perhaps the levels would not be robust enough to induce tumours, as compared to "active" SOX $2+$ stem cells targeted by oncogenic mutation. This indirect paracrine function of stem cells is of relevance to human pituitary tumour formation, as this mouse model recapitulates the most common paediatric pituitary tumour, adamantinomatous craniopharyngioma, also harbouring mutations in CTNNB1 activating the WNT signalling pathway. There is evidence of cells isolated from pituitary adenomas with similarities in their in vitro capacity and gene expression to pituitary stem cells [46]. These are also defined by a side population efflux profile upon flow cytometry following Hoechst dye labelling, which can be enriched for stem cells in many tissue types, including the pituitary [47]. Definitive evidence for cells with stem-like properties isolated from adenomas has been presented through the propagation of tumour tissue following xenotransplantation $[48,49]$. The presence of 
proliferating SOX $2+$ stem cells is prominent in papillary craniopharyngioma tumours, characterised by mutations activating the MAPK pathway [50]. The deregulation of normal developmental signalling pathways may therefore activate the proliferative potential, but sustained activation may lead to tumorigenesis, likely facilitated by stem cell involvement.

We recently described the expression of components of the Hippo signalling pathway in the pituitary [51]. This pathway has been well characterised in Drosophila, but only relatively recently described in mammals [52]. It is composed of a kinase cascade that negatively regulates the key effectors YAP and TAZ, which act to promote proliferation and the stem cell state, as well as to prevent apoptosis. It is therefore unsurprising that deregulation of YAP/TAZ underlies and promotes the growth of numerous tumours and cancers [53]. Global deletion of one of the core kinases, LATS1, results in pituitary hyperplasia and hormone deficiency [54]. The impact of LATS1 deletion on YAP/TAZ in the pituitary, as well as the possible link of this pathway to tumour involvement, remains to be shown. In the murine pituitary, localisation of YAP/TAZ is in the nucleus of SOX 2 cells and not of the committed lineages (Fig. 3a). Since nuclear YAP/TAZ is associated with an active stem cell state, this pathway may be of relevance to regulation of the stem cell potential in the pituitary. Of the genes directly regulated by YAP/ TAZ, Ctgf is a target in multiple tissues [55]. Surprisingly, mRNA in situ hybridisation to detect Ctgf expression in the pituitary does not fully recapitulate Sox 2 expression, as it is mostly localised in individual cells throughout the parenchyma (Fig. 3b). Double in situ hybridisation for Ctgf and Sox2 reveals an overlap in some Ctgf-expressing cells; however, the majority of Sox2-expressing cells do not activate this YAP/TAZ target. This could suggest that Ctgf may not be a direct target of YAP/TAZ in the pitu- itary, even though similar analyses with the additional direct target Cyr61 reveal a similar expression pattern (not shown). Alternatively, YAP/TAZ may not be triggering the full transcriptional programme associated with the active stem cell state in all SOX2 cells if, for example, this activation is being inhibited. Since there is robust activity of Hippo kinases in these cells, demonstrated by the detection of phosphorylated YAP at serine residue 127, inhibition is indeed taking place [51]. Perhaps downregulating the activity of these kinases may lift the break acting on the proliferative potential of stem cells. Persistent nuclear localisation of YAP/TAZ in SOX2 cells only and detection of Yap mRNA in these cells into adulthood, as well as the Lats $^{-/-}$phenotype, are all indicative of the possibility that the potential to respond might be in place. The vast array of genetic tools available will allow us to explore the function of this pathway in pituitary stem cells and to determine its relevance to applications related to regenerative medicine and tumours. Since the Hippo/YAP/ TAZ pathway is readily druggable, the potential to precisely influence stem cell survival and proliferation through exogenous means may be facilitated [56].

Favoured by recent methodological advances, we are on the cusp of amassing sufficient knowledge of pituitary stem cell attributes, and the signalling mechanisms that influence them, to begin manipulating their function and potential in vivo. Future research will aim to reactivate, amplify, diminish, or differentiate this population in order to facilitate future treatment approaches.

\section{Acknowledgements}

We thank Ariane Sharif and Vincent Prevot for provision of adult pituitaries (King's College London REC approval reference No. LRS-15/16-2126), Christopher Lambert for critical reading of this review, and Paul Le Tissier for helpful discussions.

\section{References}

1 Andoniadou CL, et al: Sox $2^{+}$stem/progenitor cells in the adult mouse pituitary support organ homeostasis and have tumor-inducing potential. Cell Stem Cell 2013;13:433-445.

$>2$ Aguayo-Mazzucato C, Bonner-Weir S: Pancreatic $\beta$ cell regeneration as a possible therapy for diabetes. Cell Metab 2018;27:57-67.

$>3$ Gaston-Massuet C, et al: Increased Wingless (Wnt) signaling in pituitary progenitor/stem cells gives rise to pituitary tumors in mice and humans. Proc Natl Acad Sci USA 2011;108: 11482-11487.
4 Roose H, et al: Major depletion of SOX ${ }^{+}$stem cells in the adult pituitary is not restored which does not affect hormonal cell homeostasis and remodelling. Sci Rep 2017;7:16940.

5 Fu Q, Vankelecom H: Regenerative capacity of the adult pituitary: multiple mechanisms of lactotrope restoration after transgenic ablation. Stem Cells Dev 2012;21:3245-3257.

6 Waite E, et al: Different degrees of somatotroph ablation compromise pituitary growth hormone cell network structure and other pituitary endocrine cell types. Endocrinology 2010;151:234-243.
-7 Ozone C, Suga H: Functional pituitary tissue formation. Methods Mol Biol 2017;1597:5765.

8 Ozone C, et al: Functional anterior pituitary generated in self-organizing culture of human embryonic stem cells. Nat Commun 2016;7: 10351.

$\checkmark 9$ Dincer Z, et al: Specification of functional cranial placode derivatives from human pluripotent stem cells. Cell Rep 2013;5:1387-1402.

10 Suga H, et al: Self-formation of functional adenohypophysis in three-dimensional culture. Nature 2011;480:57-62. 
11 Leung AW, Kent Morest D, Li JY: Differential BMP signaling controls formation and differentiation of multipotent preplacodal ectoderm progenitors from human embryonic stem cells. Dev Biol 2013;379:208-220.

12 Zimmer B, et al: Derivation of diverse hormone-releasing pituitary cells from human pluripotent stem cells. Stem Cell Reports 2016;6:858-872.

13 Falconi G, Rossi GL: Method for placing a pituitary graft into the evacuated pituitary capsule of the hypophysectomized rat or mouse. Endocrinology 1964;75:964-967.

14 Maxwell M, et al: Functional transplantation of the rat pituitary gland. Neurosurgery 1998; 43:1157-1163.

15 Tulipan NB, Zacur HA, Allen GS: Pituitary transplantation: part 1. Successful reconstitution of pituitary-dependent hormone levels. Neurosurgery 1985;16:331-335.

16 Vuillez P, Moos F, Stoeckel ME: Immunocytochemical and ultrastructural studies on allografts of the pituitary neurointermediate lobe in the third cerebral ventricle of the rat. Cell Tissue Res 1989;255:393-404.

17 Arnold K, et al: Sox $2^{+}$adult stem and progenitor cells are important for tissue regeneration and survival of mice. Cell Stem Cell 2011;9: 317-329.

18 Jayakody SA, et al: SOX2 regulates the hypothalamic-pituitary axis at multiple levels. J Clin Invest 2012;122:3635-3646.

19 Gremeaux L, et al: Activated phenotype of the pituitary stem/progenitor cell compartment during the early-postnatal maturation phase of the gland. Stem Cells Dev 2012;21:801-813.

20 Mollard P, et al: A tridimensional view of pituitary development and function. Trends Endocrinol Metab 2012;23:261-269.

-21 Rizzoti K, Akiyama H, Lovell-Badge R: Mobilized adult pituitary stem cells contribute to endocrine regeneration in response to physiological demand. Cell Stem Cell 2013;13:419432.

22 Lepore DA, et al: Identification and enrichment of colony-forming cells from the adult murine pituitary. Exp Cell Res 2005;308:166176.

23 Andoniadou CL, et al: Identification of novel pathways involved in the pathogenesis of human adamantinomatous craniopharyngioma. Acta Neuropathol 2012;124:259-271.

24 Garcia-Lavandeira M, et al: Craniopharyngiomas express embryonic stem cell markers (SOX2, OCT4, KLF4, and SOX9) as pituitary stem cells but do not coexpress RET/GFRA3 receptors. J Clin Endocrinol Metab 2012; 97:E80-E87.

25 Weiss S, et al: Evidence for a progenitor cell population in the human pituitary. Clin Neuropathol 2009;28:309-318.
26 Kikuchi M, et al: Immunohistochemical localization of anterior pituitary hormones in S-100 protein-positive cells in the rat pituitary gland. Cell Tissue Res 2011;345:425-429.

-27 Garcia-Lavandeira M, et al: A GRFa2/Prop1/ stem (GPS) cell niche in the pituitary. PLoS One 2009; 4:e4815.

28 Chen M, et al: Coxsackievirus and adenovirus receptor-positive cells compose the putative stem/progenitor cell niches in the marginal cell layer and parenchyma of the rat anterior pituitary. Cell Tissue Res 2013;354:823-836.

29 Susa T, et al: Paired-related homeodomain proteins Prx1 and Prx2 are expressed in embryonic pituitary stem/progenitor cells and may be involved in the early stage of pituitary differentiation. J Neuroendocrinol 2012;24: 1201-1212.

30 Gleiberman AS, et al: Genetic approaches identify adult pituitary stem cells. Proc Natl Acad Sci USA 2008;105:6332-6337.

31 Krylyshkina O, et al: Nestin-immunoreactive cells in rat pituitary are neither hormonal nor typical folliculo-stellate cells. Endocrinology 2005; 146:2376-2387.

32 Pérez Millán MI, et al: PROP1 triggers epithelial-mesenchymal transition-like process in pituitary stem cells. Elife 2016;5:e14470.

-33 Jaitin DA, et al: Massively parallel single-cell RNA-seq for marker-free decomposition of tissues into cell types. Science 2014;343:776779 .

34 Chen J, et al: Spatial transcriptomic analysis of cryosectioned tissue samples with Geo-seq. Nat Protoc 2017;12:566-580.

- 35 Lovatt D, et al: Transcriptome in vivo analysis (TIVA) of spatially defined single cells in live tissue. Nat Methods 2014;11:190-196.

- 36 Lee JH, et al: Highly multiplexed subcellular RNA sequencing in situ. Science 2014;343: 1360-1363.

37 Livet J, et al: Transgenic strategies for combinatorial expression of fluorescent proteins in the nervous system. Nature 2007;450:56-62.

38 Snippert HJ, et al: Intestinal crypt homeostasis results from neutral competition between symmetrically dividing Lgr5 stem cells. Cell 2010;143:134-144.

39 Carbajo-Pérez E, Watanabe YG: Cellular proliferation in the anterior pituitary of the rat during the postnatal period. Cell Tissue Res 1990;261:333-338.

40 Taniguchi Y, et al: Mitoses of thyrotrophs contribute to the proliferation of the rat pituitary gland during the early postnatal period. Anat Embryol (Berl) 2002;206:67-72.

41 Willems C, et al: Regeneration in the pituitary after cell-ablation injury: time-related aspects and molecular analysis. Endocrinology 2016; 157:705-721.
42 Langlais D, et al: Adult pituitary cell maintenance: lineage-specific contribution of selfduplication. Mol Endocrinol 2013;27:11031112.

43 Yin P, Kawashima K, Arita J: Direct actions of estradiol on the anterior pituitary gland are required for hypothalamus-dependent lactotrope proliferation and secretory surges of luteinizing hormone but not of prolactin in female rats. Neuroendocrinology 2002;75:392401.

44 Clevers H, Loh KM, Nusse R: Stem cell signaling. An integral program for tissue renewal and regeneration: Wnt signaling and stem cell control. Science 2014;346:1248012.

45 Gonzalez-Meljem JM, et al: Stem cell senescence drives age-attenuated induction of pituitary tumours in mouse models of paediatric craniopharyngioma. Nat Commun 2017; $8: 1819$.

46 Mertens F, et al: Pituitary tumors contain a side population with tumor stem cell-associated characteristics. Endocr Relat Cancer 2015;22:481-504.

-47 Chen J, et al: Pituitary progenitor cells tracked down by side population dissection. Stem Cells 2009;27:1182-1195.

48 Xu Q, et al: Isolation of tumour stem-like cells from benign tumours. Br J Cancer 2009;101: 303-311.

49 Chen L, et al: Evidence of brain tumor stem progenitor-like cells with low proliferative capacity in human benign pituitary adenoma. Cancer Lett 2014;349:61-66.

50 Haston S, et al: MAPK pathway control of stem cell proliferation and differentiation in the embryonic pituitary provides insights into the pathogenesis of papillary craniopharyngioma. Development 2017;144:2141-2152.

51 Lodge EJ, et al: Expression analysis of the Hippo cascade indicates a role in pituitary stem cell development. Front Physiol 2016;7:114.

52 Zhao B, Tumaneng K, Guan KL: The Hippo pathway in organ size control, tissue regeneration and stem cell self-renewal. Nat Cell Biol 2011;13:877-883.

53 Zanconato F, Cordenonsi M, Piccolo S: YAP/ TAZ at the roots of cancer. Cancer Cell 2016; 29:783-803.

54 St John MA, et al: Mice deficient of Lats1 develop soft-tissue sarcomas, ovarian tumours and pituitary dysfunction. Nat Genet 1999;21: 182-186.

55 Zhao B, et al: TEAD mediates YAP-dependent gene induction and growth control. Genes Dev 2008;22:1962-1971.

56 Johnson R, Halder G: The two faces of Hippo: targeting the Hippo pathway for regenerative medicine and cancer treatment. Nat Rev Drug Discov 2014;13:63-79. 